\title{
CSF-1 signals directly to renal tubular epithelial cells to mediate repair in mice
}

\author{
Julia Menke, ${ }^{1}$ Yasunori Iwata, ${ }^{1}$ Whitney A. Rabacal, ${ }^{1}$ Ranu Basu, ${ }^{2}$ \\ Yee G. Yeung, ${ }^{2}$ Benjamin D. Humphreys, ${ }^{1}$ Takashi Wada, ${ }^{3}$ \\ Andreas Schwarting, ${ }^{4}$ E. Richard Stanley, ${ }^{2}$ and Vicki R. Kelley ${ }^{1}$
}

\begin{abstract}
1Laboratory of Molecular Autoimmune Disease, Renal Division, Department of Medicine, Brigham and Women's Hospital, Boston, Massachusetts, USA. ${ }^{2}$ Department of Developmental and Molecular Biology, Albert Einstein College of Medicine, New York, New York, USA. ${ }^{3}$ Department of Laboratory Medicine, Institute of Medical Pharmaceutical and Health Sciences, Faculty of Medicine, Kanazawa University, Kanazawa, Japan. ${ }^{4}$ Department of Nephrology and Rheumatology, Johannes-Gutenberg University, Mainz, Germany.
\end{abstract}

\begin{abstract}
Tubular damage following ischemic renal injury is often reversible, and tubular epithelial cell (TEC) proliferation is a hallmark of tubular repair. Macrophages have been implicated in tissue repair, and CSF-1, the principal macrophage growth factor, is expressed by TECs. We therefore tested the hypothesis that CSF-1 is central to tubular repair using an acute renal injury and repair model, ischemia/reperfusion (I/R). Mice injected with CSF-1 following I/R exhibited hastened healing, as evidenced by decreased tubular pathology, reduced fibrosis, and improved renal function. Notably, CSF-1 treatment increased TEC proliferation and reduced TEC apoptosis. Moreover, administration of a CSF-1 receptor-specific (CSF-1R-specific) antibody after I/R increased tubular pathology and fibrosis, suppressed TEC proliferation, and heightened TEC apoptosis. To determine the contribution of macrophages to CSF-1-dependent renal repair, we assessed the effect

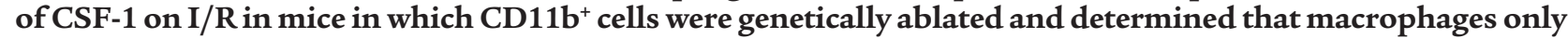
partially accounted for CSF-1-dependent tubular repair. We found that TECs expressed the CSF-1R and that this receptor was upregulated and coexpressed with CSF-1 in TECs following renal injury in mice and humans. Furthermore, signaling via the CSF-1R stimulated proliferation and reduced apoptosis in human and mouse TECs. Taken together, these data suggest that CSF-1 mediates renal repair by both a macrophage-dependent mechanism and direct autocrine/paracrine action on TECs.
\end{abstract}

\section{Introduction}

The kidney possesses a remarkable capacity to regenerate. Following acute kidney injury and subsequent inflammation, the injured kidney often heals (1). As inflammation and repair are closely intertwined, renal inflammation sets the stage for a dynamic repair process (2). Repair begins during the early phases of inflammation but only reaches completion after the injurious influence is eliminated (3). Although the pathogenesis of acute kidney injury is in part dependent on the etiology, ischemia is often a consistent feature (reviewed in ref. 4). Tubules are notably sensitive to acute renal ischemia. The tubules in the outer stripe of the outer medulla are most susceptible to ischemic injury (5). The mouse model of ischemia/ reperfusion $(I / R)$ is a particularly powerful tool for identifying the molecular mechanisms central to repair. When acute kidney injury is sufficiently severe, it leads to morbidity and mortality. Although acute renal injury is common, there are few therapeutic approaches (6). Therefore, deciphering the mechanisms that promote renal healing and in particular tubular repair using I/R in mice may offer the promise of new therapeutic strategies for acute renal injury.

There are several possible mechanisms that may be responsible for tubular repair following injury. These include bone marrow

Conflict of interest: Vicki R. Kelley received income and research support from Genzyme. She also owns stocks from Biogen, Vertex, Merck, Novartis, and Genzyme. All other authors have declared that no conflict of interest exists.

Nonstandard abbreviations used: B6, C57BL/6; BUN, blood urea nitrogen; CSF-1R, CSF-1 receptor; DT, diphtheria toxin; I/R, ischemia/reperfusion; KIM-1, kidney injury molecule 1; MTT, 3-(4,5-dimethylthiazol 1-2)-2,5-diphenyltetrazolium bromide; TEC, tubular epithelial cell; TPA, phorbol 12-mayristate $31 \mathrm{c}$ acetate; UUO, unilateral ureter obstruction.

Citation for this article: J. Clin. Invest. 119:2330-2342 (2009). doi:10.1172/JCI39087. cells directly replacing damaged tubules and intrinsic tubular epithelial cells (TECs) replenishing damaged tubules. We can rule out bone marrow cells directly replacing damaged tubules, as restoration of TECs during the repair of postischemic kidneys occurs independently of bone marrow-derived stem cells (7). Moreover, genetic fate-mapping techniques indicate that surviving TECs proliferate and replenish necrotic and apoptotic tubules following ischemic tubular injury (1). In fact, TEC proliferation is a hallmark of renal repair. For healing to advance, accompanying the replenishment of TECs, simultaneous mechanisms must halt further TEC destruction. Thus, the molecular pathways that promote TEC proliferation and halt TEC destruction following renal injury need to be elucidated.

Macrophages are instrumental in inflammation. CSF-1 is a hematopoietic growth factor that binds to a sole receptor, CSF-1 receptor (CSF-1R), expressed on macrophages $(8,9)$. During renal inflammation (lupus nephritis, unilateral ureter obstruction [UUO]), CSF-1 is generated predominantly by TECs $(10,11)$. Using mutant mice lacking CSF-1, we established that CSF-1 promotes macrophage-dependent renal inflammation (10). CSF-1 fosters the intrarenal recruitment, proliferation, and activation of macrophages that in turn release mediators responsible for inducing apoptosis of TECs (10). Thus, CSF-1 fuels the intrarenal accumulation of macrophages that are central to initiating renal inflammation. However, these findings are limited to models of inflammation in which the offending influence (autoimmune disease, obstruction) is not eliminated; thus, inflammation escalates and healing is prevented. In contrast, in a setting of ischemia-induced heart failure, autologous engraftment of CSF-1-transfected myoblasts improved the repair of the 
failing myocardium (12). This suggests that when the offending influence is transient, CSF-1 fosters tissue repair.

Nonhematopoietic and hematopoietic cells may contribute to CSF-1-dependent tissue repair. Growing evidence suggests that tissue repair is mediated by macrophages, as these CSF-1R-bearing cells switch their phenotype from tissue destroyers to regenerators (reviewed in refs. 13, 14). While macrophages dependent on CSF-1 are implicated in renal repair, the contribution of parenchymal cells to CSF-1-dependent renal repair has not been considered. Evidence suggests that epithelial and other cells may be regulated by CSF-1 in an autocrine or paracrine manner. Akin to TECs in the inflamed kidney, mammary epithelial cells express CSF-1, and during pregnancy, they also express the CSF-1R (15). Moreover, signaling via the CSF-1R stimulates proliferation of epithelial carcinoma cells and several other cell types (macrophages, myoblasts) and is central to breast remodeling during pregnancy $(16,17)$. This raises the possibility that CSF-1R expression on CSF-1-expressing TECs mediates tubular repair. We hypothesized that CSF-1 hastens renal repair in a model of renal injury with a transient insult, I/R. Furthermore, we hypothesized that CSF-1-dependent renal repair is only partially mediated by macrophages and that a macrophage-independent autocrine/ paracrine mechanism leads to renal tubular repair.

We now report that administration of CSF-1 after renal injury fosters improved renal tubular pathology and renal function, decreases renal fibrosis, accelerates the rate of tubular proliferation, and suppresses tubular apoptosis. Genetic ablation on macrophages revealed that macrophages are only partially responsible for CSF-1-dependent renal repair. Investigating whether CSF-1 signaling on TEC directly mediates renal repair, we determined that TECs express the CSF-1R and that this receptor is upregulated and coexpressed with CSF-1 on TEC during acute renal injury in mice and humans. Signaling via this CSF-1R on TEC stimulates TEC proliferation and dampens TEC apoptosis. Thus, we have identified a CSF-1-dependent, macrophage-independent autocrine/paracrine mechanism in tubules that is instrumental in tubular self repair following acute renal injury.

\section{Results}

CSF-1 mediates repair during $I / R$. Since CSF- 1 is instrumental in renal inflammation $(10,18-20)$ and inflammation sets the stage for repair, we hypothesized that CSF-1 promotes kidney repair. To test this hypothesis, we used a model of renal ischemic injury and repair. Tubules in the outer medulla are notably sensitive to ischemia and are readily damaged, often with sufficient severity to result in death. However, the kidney is resilient, and most often the damaged tubules regenerate and the kidney repairs. This process is rapid and the pathology peaks by 2-3 days; repair is evident by 5 days following I/R (Supplemental Figure 1A; supplemental material available online with this article; doi:10.1172/JCI39087DS1). To determine whether CSF-1 accelerates healing, we selected a time point to evaluate mice following $\mathrm{I} / \mathrm{R}$ at which repair is evident but incomplete ( 5 days). Using this model, we injected CSF-1 (every 12 hours) after I/R (1.5-4.5 days) and evaluated kidneys for tubular pathology, renal function, and fibrosis (collagen) in comparison with age- and sex-matched PBS-injected mice at 5 days following I/R. CSF-1-injected mice had less tubular pathology (dilated tubules, casts) in the outer medulla and cortex as compared with PBS-injected controls (Figure 1A). Consistent with this finding, we detected less fibrosis in the kidney and better renal function (albu- minuria, blood urea nitrogen [BUN]) in mice injected with CSF-1 compared with those injected with PBS (Figure 1A). Moreover, we detected a decrease in kidney injury molecule 1 (KIM-1), a molecule whose level of expression directly correlates with the degree of TEC injury (21), in mice injected with CSF-1 compared with those injected with PBS (Supplemental Figure 2). Based on this finding, we hypothesized that blocking the CSF-1R following I/R delays renal repair. To test this hypothesis, we compared mice injected with anti-CSF-1R Abs instead of CSF-1 with age/sex/strain/procedure-matched mice injected with control Abs (rat IgG) during the same time frame following I/R. We detected increased tubular pathology, more fibrosis, a greater impairment of renal function (albuminuria; Figure 1B), and an increase in KIM-1 expression in TECs (Supplemental Figure 2) in mice injected with anti-CSF-1R Abs compared with those injected with rat IgG (Figure 1B). This suggests that CSF-1 mediates renal repair following I/R.

CSF-1 promotes TEC Proliferation andreduces TEC apoptosis following $I / R$. TEC proliferation is a hallmark of renal repair (22). Moreover, reducing TEC apoptosis is necessary for healing. Since CSF-1 mediates renal repair, we hypothesized that CSF-1 promotes TEC proliferation and reduces TEC apoptosis following I/R. TEC proliferation reaches an apex on day 3 , is on the decline by day 5 (Figure $1, A$ and B), and nearly returns to baseline at day 7 (Supplemental Figure 1B). Using the same mice examined above, we detected an amplified rise (day 3 ) and a more abrupt decline (day 5) in tubular proliferation in mice injected with CSF-1 compared with those injected with PBS following I/R (Figure 1A). This suggests that CSF-1 promotes tubular proliferation after I/R. Moreover, tubular apoptosis in mice injected with CSF-1 compared with those injected with PBS diminished following I/R (day 5 ). To verify that CSF-1 promotes TEC proliferation and reduces TEC apoptosis, we blocked the CSF-1R after I/R. Blocking the CSF-1R suppressed TEC proliferation (days 3 and 5) and heightened TEC apoptosis (day 5) (Figure 1B). This suggests that CSF-1 mediates renal repair by promoting TEC proliferation and reducing TEC apoptosis.

The CSF-1R is expressed on TECs and upregulated following in vitro stimulation with CSF-1. Since the CSF-1R is expressed on mammary epithelial and other nonmacrophage cell types $(16,17)$, it was possible that TECs also express the CSF-1R. Therefore, we determined CSF-1R transcript and protein expression using cultured TECs, first focusing on mouse TECs. We identified CSF-1R mRNA transcripts in primary cultured mouse TECs and the $\mathrm{C} 1$ proximal TEC cell line (Figure 2A). In comparison, T cells (DO 11.10 line) did not express CSF-1R mRNA. To verify CSF-1R expression, we used primary TECs derived from $C s f 1 R^{-/-}$mice as a negative control. Of note, although TECs expressed CSF-1R transcripts, the level of expression was far less than in macrophages (primary cultured BM macrophages and RAW line). To determine whether CSF-1 upregulates the expression of CSF-1R transcripts on TECs, we incubated the C1 TEC line with CSF-1. CSF-1 stimulation increased CSF-1R transcripts by 24 hours, and expression rose even higher by 48 hours (Figure 2A). T cells (DO11.10 line) and macrophages (RAW line) served as negative and positive controls, respectively. To establish that the CSF-1R protein is expressed on TECs, we determined its expression by immunofluorescence staining and by Western blotting. The CSF-1R was readily detected on primary cultured TECs by immunofluorescence using anti-CSF-1R Abs (Figure 2A). We confirmed the specificity of this Ab by using WT $\mathrm{BM}$ macrophages as a positive control and primary cultured TECs derived from $C s f 1 R^{-/-}$mice as a negative control. Expression of 
A

Ischemia/Reperfusion (I/R)

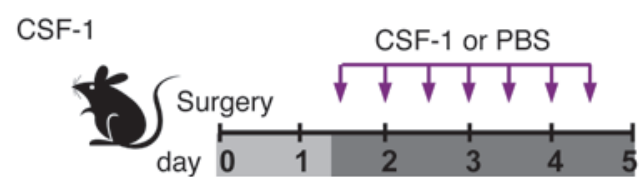

B Tubules:

Histopathology
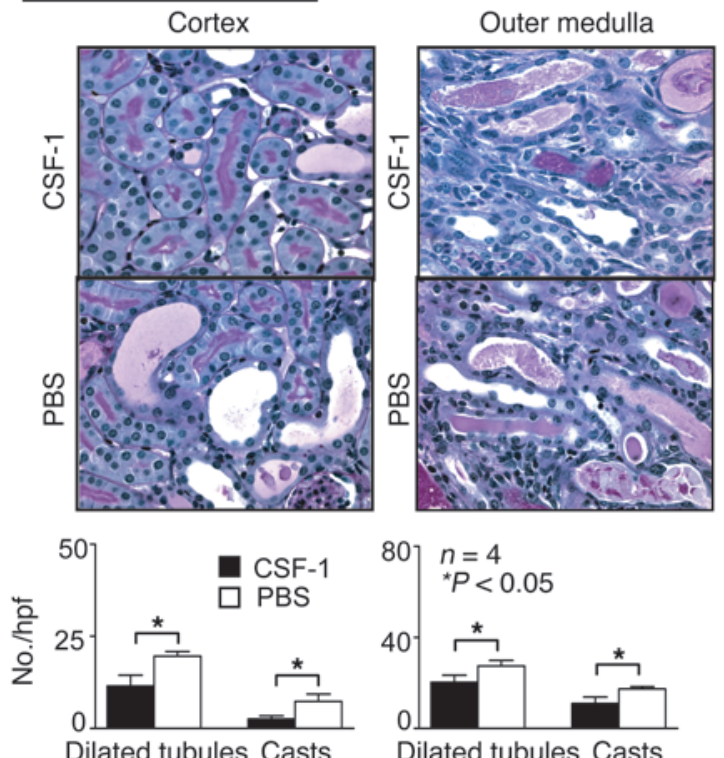

C Fibrosis

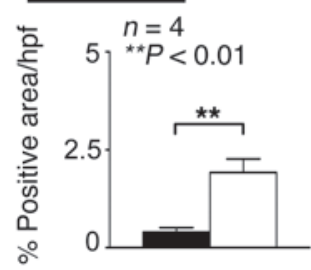

D Renal function

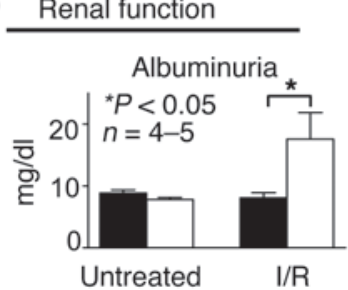

E Tubules:

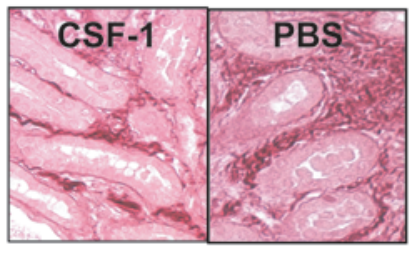

CSF-1 BUN $\square$ PBS
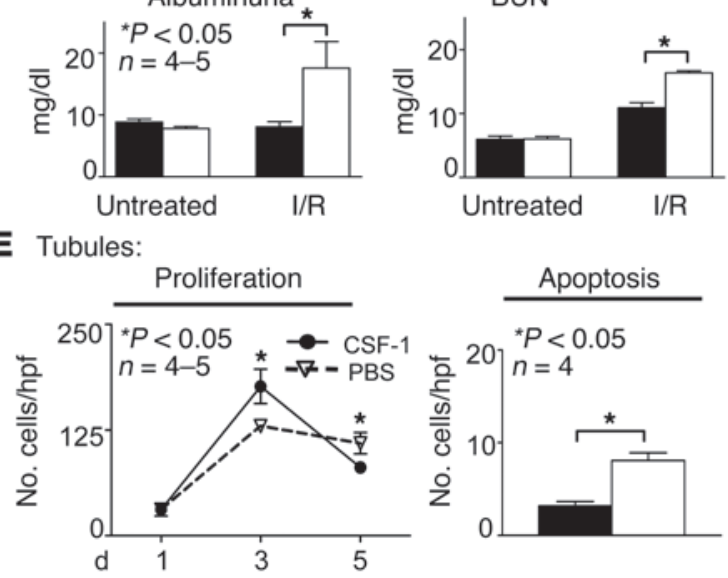

CSF-1R-Ab

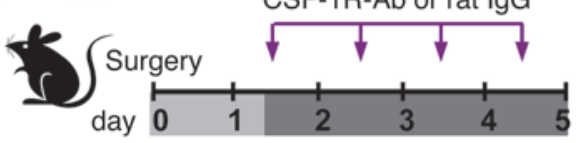

Tubules:

Histopathology
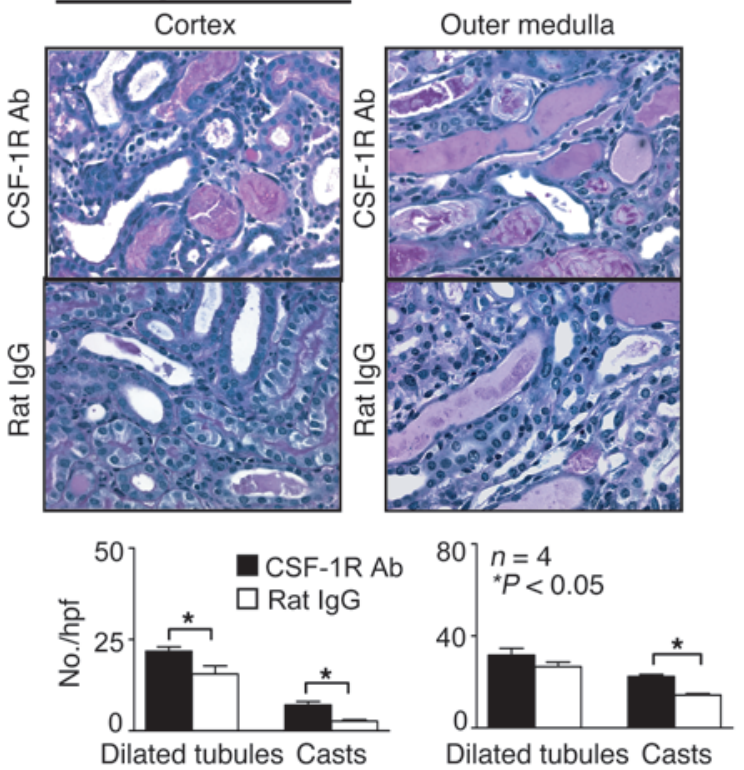

Fibrosis
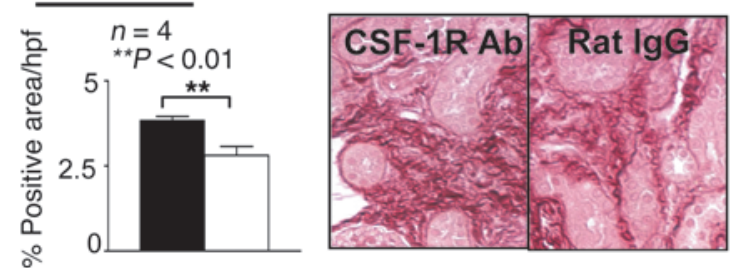

- CSF-1R Ab BUN $\square$ Rat IgG
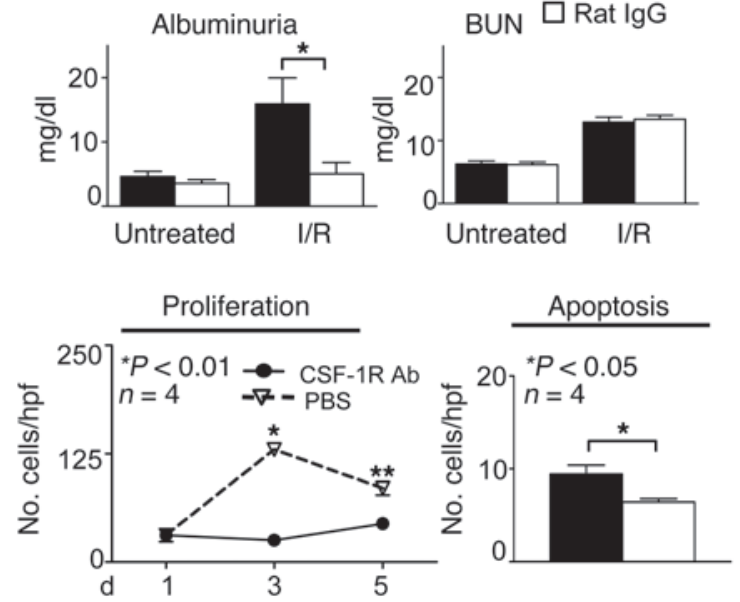


\section{Figure 1}

CSF-1 hastens kidney repair and blocking CSF-1R hinders kidney repair following I/R. (A) Left panel: B6 mice were injected twice daily with either CSF-1 or PBS starting at day 1.5 following I/R until day 4.5. Right panel: B6 mice were injected daily with either CSF-1R or an irrelevant control $\mathrm{Ab}$ (rat $\operatorname{lgG}$ ) starting at day 1.5 following I/R until day 4.5. Except for those shown in part $\mathbf{E}$, all mice were sacrificed for analysis on day 5 following I/R. (B) Left panel: renal tubular histopathology (cortex and outer medulla) was less severe in CSF-1-injected than in PBS-injected mice. Right panel: renal tubular histopathology was more severe in CSF-1R Ab-injected than in control Ab-injected mice. Representative photomicrographs and corresponding graphs indicate the number of dilated tubules and casts in the cortex and outer medulla. (C) Left panel: fibrosis (collagen staining, sirius red) of TECs is decreased in mice receiving CSF-1 compared with those receiving PBS. Right panel: fibrosis is increased in CSF-1R Ab-injected compared with control Ab-injected mice. (D) Left panel: renal function (albuminuria, BUN) is attenuated following I/R in CSF-1-treated compared with PBS-treated B6 mice. Right panel: CSF-1R Ab treatment resulted in a rise of albuminuria. (E) Left panel: mice injected with CSF-1 compared with PBS sacrificed on days 1,3 , and 5 following $\mathrm{I} / \mathrm{R}$ revealed that TEC proliferation in CSF-1-injected mice peaks and declines more rapidly than in PBS-injected mice. The number of apoptotic TECs evaluated by immunostaining for cleaved caspases-3 decreased in mice receiving CSF-1 compared with those receiving PBS. Right panel: TEC proliferation is reduced while the number of apoptotic cells is increased in CSF-1R Ab-injected compared with control Ab-injected mice. Representative photomicrographs are shown. Data show means \pm SEM. Original magnification, $\times 40$.

bands corresponding to both the $135-\mathrm{kDa}$ CSF-1R precursor and the mature $165-\mathrm{kDa}$ CSF-1R was also shown in primary cultured TECs (C57BL/6 [B6]) and control primary BM macrophages by Western blotting (Figure 2A). CSF-1R expression was 3-fold lower in the TECs than in the BM macrophages. Thus, the CSF-1R is expressed on mouse TECs.

To determine whether human TECs express the CSF-1R, we examined CSF-1R mRNA and protein expression in the human HK2 TEC line. HK2 cells express CSF-1R mRNA transcripts (Figure 2B). Consistent with our findings in mice (Figure 2A), T cells (Jurkat) did not express CSF-1, and the level of CSF-1R expression on macrophages (U937, K562, and HL60 lines stimulated with phorbol 12-mayristate $31 \mathrm{c}$ acetate [TPA]) was far more robust than on TECs (Figure 2B). Similarly, we detected CSF-1R protein in human HK2 TEC lysates by Western blotting (Figure 2B). The data indicate that both mouse and human TECs express the CSF-1R, albeit at lower levels than in macrophages.

Macrophages only partially account for CSF-1-dependent renal repair after $I / R$. Our results thus far indicate that CSF- 1 mediates renal repair after $\mathrm{I} / \mathrm{R}$ by promoting TEC proliferation and reducing TEC apoptosis and that TECs express the CSF-1R. Since macrophages express the CSF-1R and have been implicated in renal repair, it is important to determine the contribution of macrophages to CSF-1-mediated repair following injury. Since growing evidence indicates that a single macrophage marker does not exclusively identify macrophages (23), we have used 2 markers to identify macrophages, CD68 and F4/80. Using the same proto$\mathrm{col}$ as depicted in Figure 1, we detected an increase in intrarenal macrophages in mice injected with CSF-1 as compared with those injected with the vehicle control after I/R (Supplemental Figure $3)$. The increase in intrarenal macrophages was selective, since in contrast, intrarenal $\mathrm{T}$ cells decreased (data not shown). Moreover, we detected a decrease in intrarenal macrophages in mice injected with anti-CSF-1R Abs as compared with the IgG control after I/R (Supplemental Figure 3).

To determine the contribution of macrophages to CSF-1dependent renal repair, we used CD11b-DTR mice with a diphtheria toxin-inducible (DT-inducible) system that transiently depletes macrophages (24). Following the injection of DT into CD11b-DTR mice at days 1 and 3 after I/R, we evaluated macrophages at days 3 and 5 . At day 5 , the number of intrarenal macrophages in CD11b-DTR mice injected with DT was reduced to the levels in sham controls (Supplemental Figure 4). In comparison, WT mice injected with DT had a robust number of macrophages in the kidney after I/R. Since DT markedly ablates macrophages in tissues by 12 hours and they are not completely restored until 4 days (24), we are confident that macrophages are eliminated between 1.5 and 5 days after I/R using our DT treatment protocol and that the CD11b-DTR mice can be used to evaluate the macrophage contributions. Therefore, we compared the impact of CSF-1 on renal repair in macrophage-deficient DT-treated mice (response of TECs alone), WT mice (response of macrophages and TECs), and WT mice injected with anti-CSF-1 Abs (no response of TECs or macrophages). We determined that blocking CSF-1 on TECs and macrophages hindered CSF-1-dependent renal tubular repair far more than eliminating macrophages alone (Figure 3). While albuminuria was higher in mice without macrophages as compared with those with macrophages, blocking the CSF-1R resulted in a further rise (Figure 3C). Similarly, while fibrosis increased in mice without macrophages, blocking the CSF-1R resulted in even more fibrosis (Figure 3D). Strikingly, while CSF-1-dependent tubular proliferation decreased at 3 and 5 days after $\mathrm{I} / \mathrm{R}$ in mice without macrophages, blocking the CSF-1R reduced proliferation substantially further, nearly to baseline (Figure 3E). Although CSF-1-dependent TEC apoptosis increased after eliminating macrophages, blocking the CSF-1R further amplified the number of apoptotic TECs (Figure 3E). Moreover, blocking the CSF-1R increased tubular pathology as compared with that in CSF-1 treated mice with or without macrophages (Figure 3B). Interestingly, at day 5, removing macrophages alone had not created a detectable impact on tubular histopathology, despite the increase of loss of renal function and fibrosis (Figure 3C). Taken together, our data indicate that the suppression of tubular repair caused by the blocking of CSF-1R pathways in the kidney is greater than that caused by eliminating macrophages alone. This suggests that another CSF-1R-bearing cell contributes to CSF-1-dependent renal repair.

The expression of CSF-1R and CSF-1 on TECs is increased after renal injury. Since the CSF-1R is expressed by cultured TECs, we determined whether the CSF-1R is upregulated on TECs after renal injury. For this purpose, we induced renal injury using I/R and another model of renal injury, UUO, in MacGreen mice. MacGreen mice express a reporter gene for the CSF-1R (EGFP driven by the c-fms promoter) (25). CSF-1R expression (Figure 4A) increased on TECs during I/R and UUO as compared with on MacGreen sham controls, in which expression was barely detected on TECs (Figure 4A). WT mice served as negative controls. In comparison, EGFP staining was far brighter on macrophages than on TECs in I/R, $\mathrm{UUO}$, and sham controls. This is consistent with our in vitro data, indicating that while TECs express CSF-1R, expression is more robust on macrophages. Moreover, we did not detect CSF-1R on cells within glomeruli (Supplemental Figure 5). 


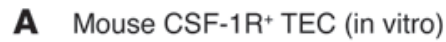
Transcripts
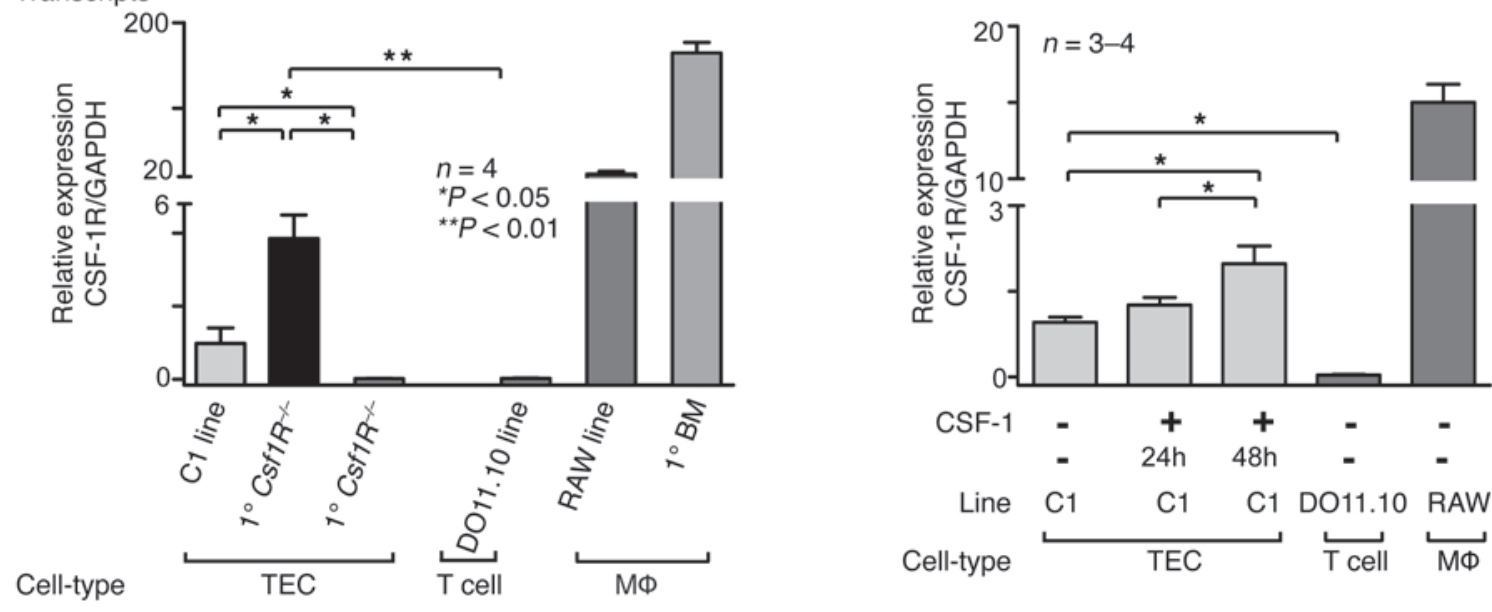

Protein
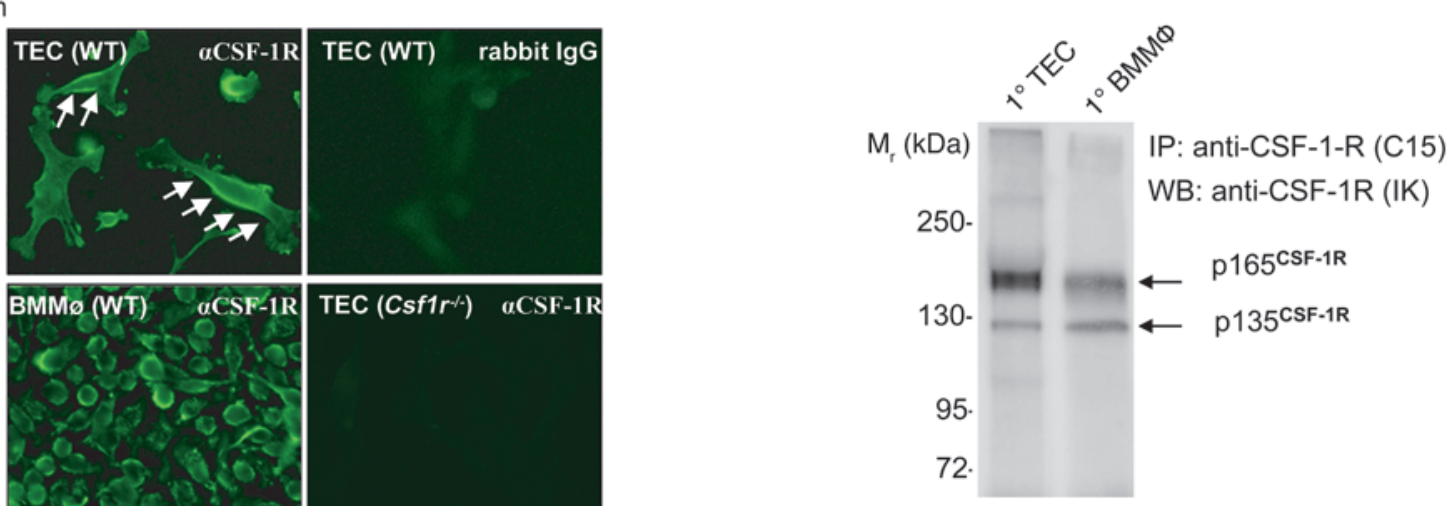

B Human CSF-1 R+ TEC (in vitro)

Transcripts

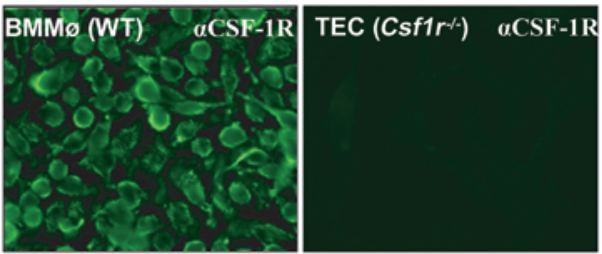

Cell-type

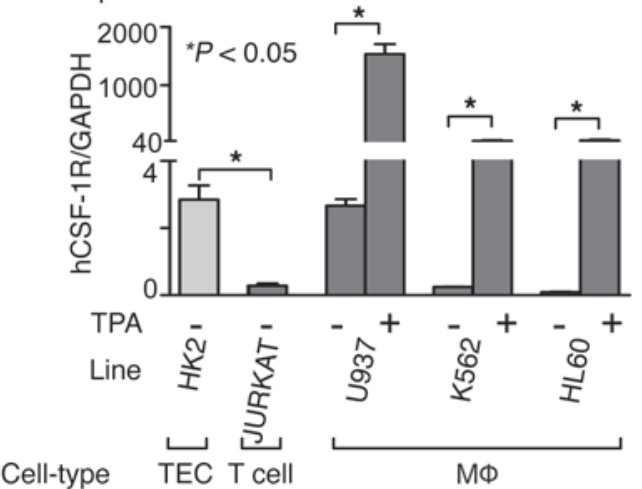

Protein

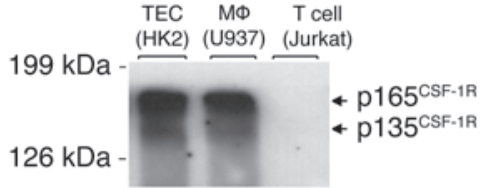




\section{Figure 2}

CSF-1R expression on mouse and human TECs. (A) Left panel: mouse TECs express CSF-1R mRNA. CSF-1R transcript expression, normalized to GAPDH expression, determined by real-time PCR in primary cultured TECs derived from WT B6 mice and in cells of the proximal TEC line, C1. TECs from Csf1 $R^{-1-}$ mice and a T cell line (DO11.10) served as negative controls, and WT BM macrophages and cells of the RAW 264 macrophage cell line served as positive controls. Right panel: CSF-1 upregulates TEC CSF-1R transcript expression. C1 cells were incubated with CSF-1 (100 ng/ml) for 24 hours and 48 hours. Results are representative of 3 separate experiments. Means \pm SEM are shown. Bottom left panel: Primary TECs express CSF-1R protein. Primary WT BM macrophages and primary Csf1 $R^{-1-}$ TECs served as positive and negative controls, respectively ( $n=2-3$ per group). Original magnification, $\times 40$. Bottom right panel: CSF-1R protein detected in lysates of cultured primary WT TECs. Western blot of CSF-1R immunoprecipitates. WT BM macrophages (at less than one-third the amount of TEC protein) served as a positive control. (B) Left panel: CSF-1R transcript expression in cultured cells of the human proximal TEC line, HK2, determined by real-time PCR. The Jurkat T cell line, the human leukemic monocytic leukemia (U937), the human erythromyeloblastoid leukemia cell line (K562), and the human leukemic cell line (HL60), all stimulated with TPA, served as positive controls. Results are representative of 3 separate experiments. Data represent means \pm SEM. Right panel: Human TECs (HK2 line) express CSF-1R protein detected by Western blotting. TPA-stimulated U937 cells and Jurkat T cells served as positive and negative controls, respectively.

We previously reported that CSF-1 expression in TECs is upregulated during renal inflammation (26). To determine whether CSF-1 and CSF-1R expression is simultaneously enhanced on TECs following renal injury (I/R, UUO), we examined the expression of CSF-1 using TgZ CSF-1 reporter mice (27), which express lacZ under the control of the CSF-1 promoter/first intron. CSF-1 expression, indicated by $\beta$-gal staining, was apparent on TECs in TgZ mice after I/R and UUO at the same time as CSF-1R expression (Figure 4B). In comparison, low levels of $\beta$-gal staining were detected in sham controls (Figure 4B). Thus, CSF-1 and CSF-1R were coexpressed on TECs after I/R and UUO. In comparison, CSF-1 was sparsely expressed by cells in glomeruli with a mesangial and podocyte distribution (Supplemental Figure 5). Since CSF-1 upregulates CSF-1R in cultured TECs (Figure 2A), it is possible that CSF-1 expressed by TECs may enhance CSF-1R expression on TECs in response to renal injury in mice.

CSF-1 induces survival/proliferation in TECs after renal injury. The expression of both CSF-1 and the CSF-1R are upregulated on TECs after renal injury. Since injecting CSF-1 enhances TEC proliferation after renal injury, we hypothesized that renal injury induces CSF-1-dependent autocrine TEC proliferation. To test this hypothesis, we first stimulated human HK2 TECs with increasing concentrations of CSF-1 and evaluated proliferation by 3-(4,5-dimethylthiazol 1-2)-2,5-diphenyltetrazolium bromide (MTT) assay. TEC proliferation rose with increasing concentrations of CSF-1 $(0,12,36 \mathrm{ng} / \mathrm{ml})$, attaining maximum cell density at $36 \mathrm{mg} / \mathrm{ml}$ (Figure 5A). As expected, blocking the CSF-1R suppressed CSF-1-stimulated TEC proliferation (Figure 5A). This result indicates that $\mathrm{CSF}-1$ can stimulate the proliferation of cultured human TECs.

To establish that injured TECs express CSF-1 that, in turn, promotes TEC proliferation/survival, we incubated human HK2 TECs with actinomycin D, a compound known to injure TECs, and measured both supernatant CSF-1 and the TEC cell mass. Exposing
TECs to increasing concentrations of actinomycin D resulted in a progressive increase in CSF-1 released into the supernatant (Figure $5 B)$. Actinomycin D treatment $(0.02-0.12 \mu \mathrm{g} / \mathrm{ml})$ reduced cell numbers, but the residual cell mass was proportional to the amount of CSF-1 released in response to actinomycin D and was reduced to a common low value in cultures containing anti-CSF-1R Abs (Figure 5B). We detected similar results to these in human HK2 TECs recovering from injury induced by TNF- $\alpha / L P S$ or cisplatin (Supplemental Figure 6A). We repeated these experiments with primary cultured mouse (B6) TECs with comparable findings (Supplemental Figure 6, B-D). Furthermore, to exclude the impact of genetic background, we repeated these studies using primary cultured TECs from C3H/Fej mice and the results were similar (data not shown). Taken together, these experiments indicate that cultured TECs proliferate in response to CSF-1 and generate CSF-1 in response to injury that could contribute to their CSF-1Rdependent survival/proliferation following injury.

To determine whether CSF-1 and CSF-1R are coexpressed on tubules that proliferate, we evaluated sequential kidney sections from patients with tubular injury and impaired renal function after kidney transplantation. CSF-1 and CSF-1R expression in tubules was highly correlated, and the tubular expression of CSF-1 correlated with the percentage of proliferating $\mathrm{Ki} 67^{+} \mathrm{TECs}$ within CSF- $1^{+}$tubules in these patients (Figure 5C). Tubules containing proliferating TECs were those expressing CSF-1 and CSF-1R (Figure $5 \mathrm{C}$ ). We verified specificity of the CSF-1 and CSF-1R Abs by preabsorbing them with their respective blocking peptides and abolishing staining (Figure 5C). In contrast, tubules from normal individuals barely expressed either CSF-1 or the CSF-1R and were not proliferating (data not shown). Moreover, we pinpointed the CSF-1 and CSF-1R expression within the distal and proximal TECs and loops of Henle based on location and morphology. In contrast, we detected sparse CSF-1 expression in glomeruli (mesangial and podocyte distribution), and CSF-1R expression was even more sparse and on only a few glomeruli (Supplemental Figure 7). Importantly, we determined that CSF-1 $\mathrm{R}^{+}$tubules expressed the tyrosine-phosphorylated CSF-1R (Figure 5D). Taken together, our results thus far indicate that TEC survival/proliferation is, at least in part, regulated by CSF-1 in an autocrine/paracrine manner. Thus, tubular coexpression of CSF- 1 and CSF-1R on TECs is protective and promotes self replenishment of destroyed TECs.

CSF-1 suppresses TEC apoptosis. In order to more fully examine the impact of CSF-1 on survival, we determined whether CSF-1 expression in injured TECs dampens TEC apoptosis. Adding CSF-1 to TECs stimulated with TNF- $\alpha$ /LPS suppressed apoptosis of human HK2 TECs, albeit modestly (Figure 6A). Similar results were obtained using primary cultured TECs (data not shown). To verify that CSF-1 mediates suppression of TEC apoptosis after injury, we added anti-CSF-1R Abs along with CSF-1 (Figure 6). Blocking signaling via CSF-1R on TECs eliminated the antiapoptotic impact of CSF-1. Moreover, TNF- $\alpha /$ LPS-stimulated TECs expressed sufficient levels of CSF-1 to suppress TEC apoptosis, since blocking the CSF-1R increased TEC apoptosis without exogenous CSF-1. We detected comparable results using other molecules that injure human TECs (actinomycin D, cisplatin) (Supplemental Figure $8 \mathrm{~A}$ ), and the results were similar using primary human TECs (data not shown). These experiments were repeated and the results were similar using mouse primary TECs from B6 (Supplemental Figure $8 \mathrm{~B}$ ) and $\mathrm{C} 3 \mathrm{H} / \mathrm{Fej}$ mice (data not shown). Taken together, these results suggest that autocrine CSF-1-dependent TEC proliferation 
A

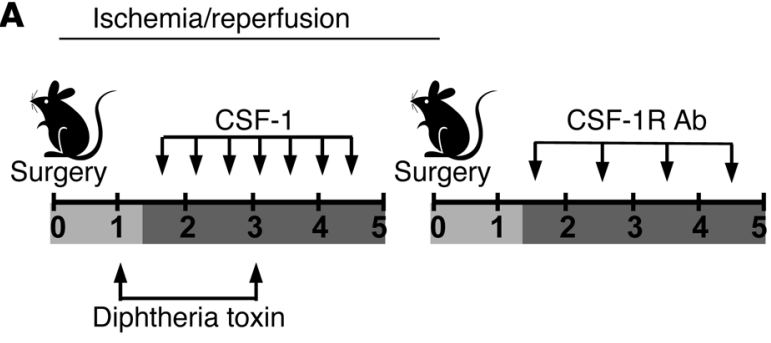

B Tubular histopathology (outer medulla)

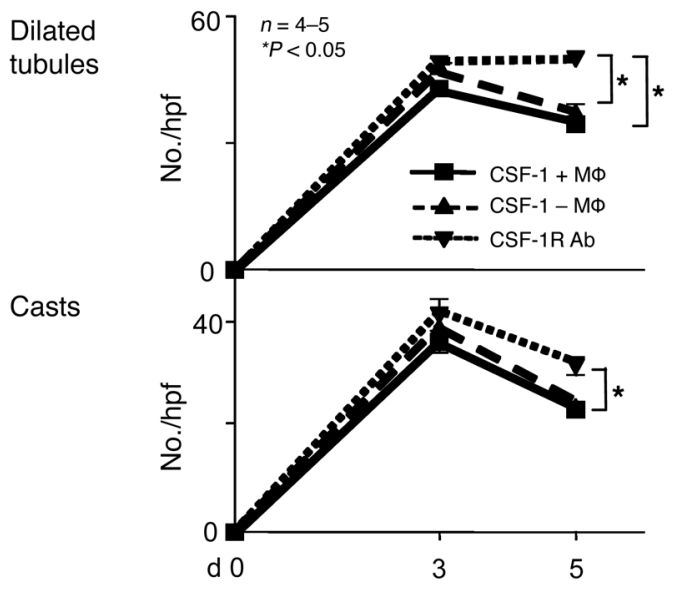

C Renal function

D Fibrosis
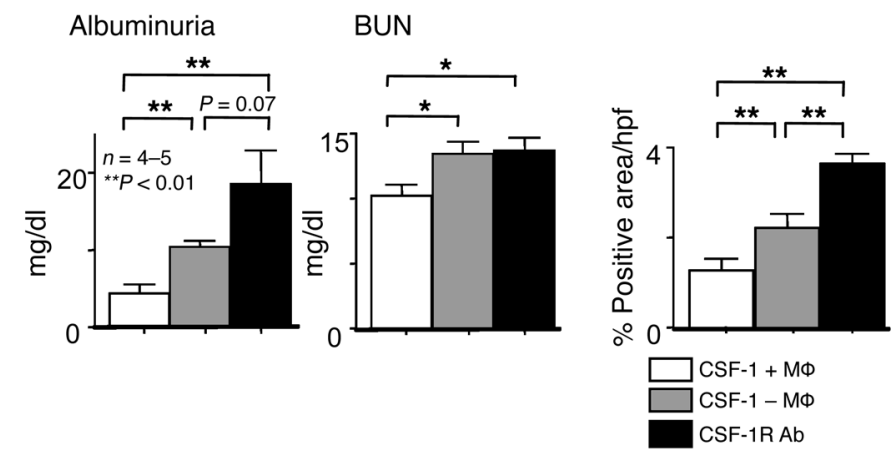

$\mathbf{E}$

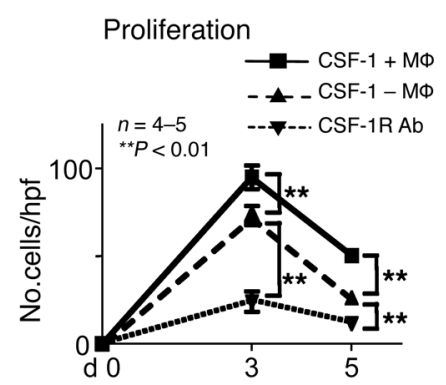

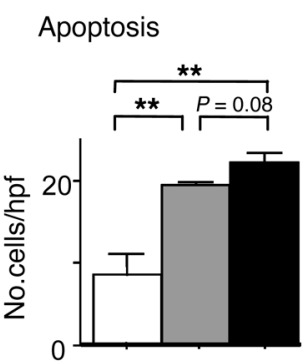

Figure 3

Macrophages are only partially responsible for CSF-1-dependent renal repair. (A) CD11b-DTR and WT mice were injected with CSF-1 following renal injury. We injected DT to deplete macrophages as indicated in the diagram, and we injected anti-CSF-1R Abs as in the diagram. (B) Tubular pathology (outer medulla) and (E) TEC proliferation were analyzed at days 0,3 , and 5 after I/R. (C) Renal function (albuminuria, BUN), (D) fibrosis (collagen), and (E) tubular apoptosis (caspase-3) were analyzed at day 5 after I/R. Data represent means \pm SEM.

and antiapoptotic mechanisms are poised to protect tubules after injury and to foster repair.

\section{Discussion}

We now report that CSF-1 promotes tubular repair following acute ischemic renal injury. Administration of CSF-1 after renal injury fosters improved renal tubular pathology and renal function, decreases renal fibrosis, accelerates the rate of tubular proliferation, and suppresses tubular apoptosis. Genetic ablation of macrophages revealed that macrophages are only partially responsible for CSF-1-dependent renal repair. We also report the finding, which we believe to be novel, that the CSF-1R is upregulated on TECs during acute renal injury in mice and humans. Moreover, signaling via CSF-1R on injured TECs is instrumental in tubular self repair. Our studies indicate that CSF-1 is generated by injured TECs and upregulates the CSF-1R on TECs. CSF-1 generated by TECs binds to the CSF-1R and in turn stimulates tubular proliferation and counters tubular apoptosis. Thus, we have identified a CSF-1-dependent, macrophage-independent autocrine/paracrine mechanism in tubules that is instrumental in tubular self repair following acute renal injury.

CSF-1 initiates renal inflammation and promotes healing. This may seem counterintuitive; however, inflammatory and repair processes are closely intertwined (2). To understand the CSF-1-dependent mechanism central to renal repair, it is critical to identify the intrarenal cells that express CSF-1 and the CSF-1R in conjunction with the type and phase of injury being explored. Our prior experimental approaches of deleting (10), overexpressing, and limiting expression to an individual CSF-1 isoform in mutant mice (26) and of local delivery of CSF-1 into the kidney using genetically modified TECs (20) established that CSF-1 promotes renal inflammation. However, these studies were limited to exploring the impact of CSF-1 in advance of renal injury in mouse models that fail to eliminate the offending agent and do not heal. In support of the beneficial impact of CSF-1 on healing, the rate of TEC repair following I/R in CSF-1-deficient (Csf1 $\left.{ }^{\circ /} /{ }^{\circ p}\right)$ compared with WT mice is reduced (data not shown). The current findings, using an acute renal injury model that leads to healing, indicate that administration of CSF-1 fosters renal repair. During the repair phase in an injured tissue, damaged tissue may be replaced by regenerating parenchymal cells and/or replacing with fibrous tissue (reviewed in ref. 28). In our I/R studies, CSF-1 promoted tubular proliferation, dampened tubular apoptosis, and lessened fibrosis. The net result was a more rapid improvement in renal tubular pathology and restoration of renal function. The major source of intrarenal CSF-1 following renal injury (26) and in particular I/R is the TECs. In comparison, the glomeruli express sparse amounts of CSF-1. Since macrophages express the CSF-1R and are abundant in the renal interstitium adjacent to TECs after $\mathrm{I} / \mathrm{R}$, it is reasonable to suggest that the invading macrophages that are recruited to CSF-1rich sites contribute to renal repair. This concept is supported by recent reports implicating macrophages in the repair of the kidney 


\section{A CSF-1 $\mathrm{R}^{+}$TEC}
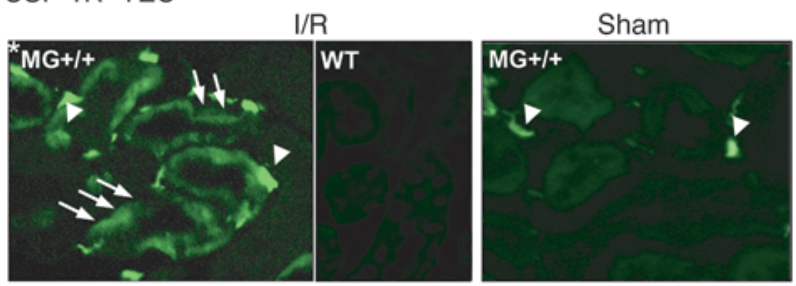

UUO
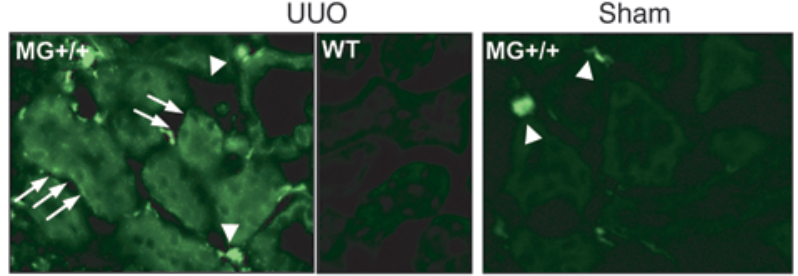

B $\mathrm{CSF}-1^{+} \mathrm{TEC}$

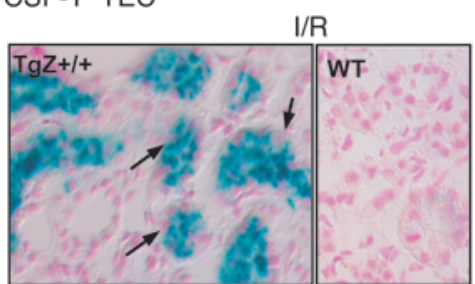

UUO

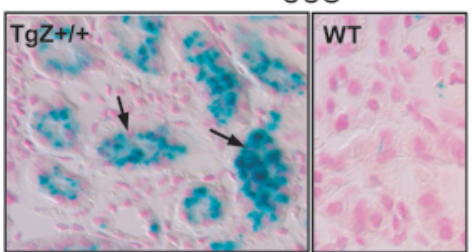

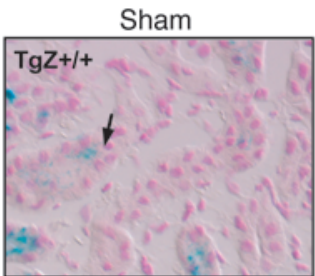

Sham

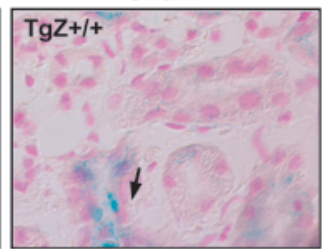

MG mice - CSF-1 $\mathrm{R}^{+}$cells
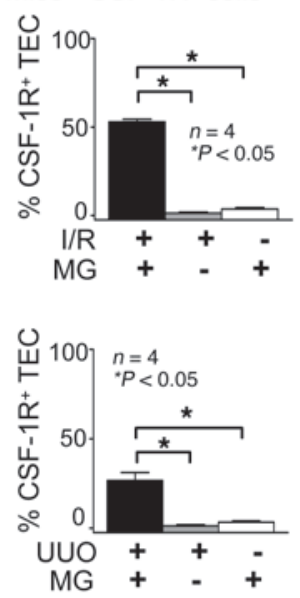

TgZ mice - CSF- $1^{+}$cells
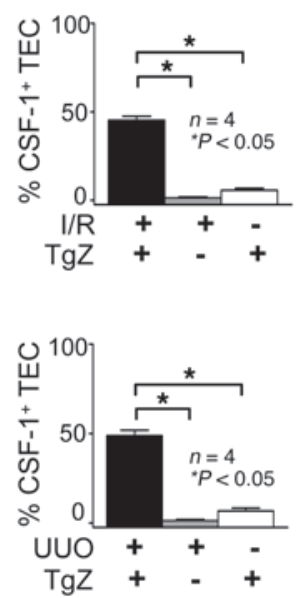

Figure 4

CSF-1R and CSF-1 expression on TECs are increased following renal injury. (A) CSF-1R gene expression is upregulated on TECs following $\mathrm{I} / \mathrm{R}$ and UUO. TECs derived from MacGreen;B6 $\left(\mathrm{MG}^{+/+} ; \mathrm{B} 6\right)$ mice have increased EGFP (arrows) 3 days after I/R or 5 days after unilateral ureter ligation (UUO) as compared with those derived from sham control and WT kidneys. Note EGFP+ macrophages in the renal interstitium (arrowheads). Original magnification, $\times 40$. Right panels: representative photomicrographs from MacGreen;B6 kidneys after I/R or UUO, together with quantitation of the percentage of EGFP+ TECs. (B) CSF-1 gene expression is upregulated on TECs following I/R and UUO. CSF-1 reporter lacZ expression by TECs in TgZ;B6 mice detected by X-gal staining 3 days after $\mathrm{I} / \mathrm{R}$ or 5 days after UUO as compared with that in sham controls and WT kidneys. Representative photomicrographs. Original magnification, $\times 40$. Right panels: quantitation of the percentage of $\beta$-gal-positive TECs. Data show means \pm SEM.
(13) and the heart (29-31). And in particular, following ischemic damage to the kidney, eliminating macrophages using lipoclodronate hinders renal repair (32). In keeping with this concept, we detected a greater accumulation of intrarenal macrophages in mice injected with CSF-1 that healed more rapidly as compared with controls. By genetically ablating macrophages, our studies indicate that macrophages only partially contribute to this CSF-1dependent renal repair. Thus, we must broaden our view of the CSF-1-dependent mechanisms leading to renal repair to include renal parenchymal cells expressing CSF-1R. We now report that TECs express low levels of CSF-1R but that, following renal injury in mice and humans, these receptors are upregulated and expression of CSF-1 is elevated. Not surprisingly, the expression of CSF-1 and CSF-1R is sparse and virtually nonexistent, respectively, in glomerular parenchymal cells. This is consistent with renal injury being largely confined to TECs rather than to glomeruli after an ischemic insult. Heightened coexpression of CSF-1 and CSF-1R on TECs is in keeping with findings in other epithelial cells. CSF-1 and CSF-1R are abundantly coexpressed on breast, ovarian, uterine, and prostate epithelial carcinomas and in mammary epithelial cells during pregnancy $(15,33,34)$. Our findings suggest that the spectrum of epithelial cells coexpressing CSF-1 and CSF-1R may expand to cells exposed to injury and inflammation. Thus, it is tempting to speculate that a CSF-1-mediated mechanism may contribute to epithelial cell repair in a broad range of tissues.

There is growing evidence that, following renal injury, tubules express multiple molecules central to self repair and self protection. Some of these pathways promote autocrine/paracrine TEC proliferation and others counter TEC apoptosis. For example, the heparin-binding EGF-like growth factor (HB-EGF) is induced in the kidney after acute tubular injury (I/R in rats) and may act as an autocrine/paracrine growth factor involved in proliferation of TECs and the subsequent repair of the kidney $(35,36)$. Macrophage-stimulating protein (MSP) and its ligand RON (37), which are weakly coexpressed on TECs and are upregulated following TEC injury, may resemble the regulation by CSF-1/CSF-1R, as in vitro, MSP stimulates TEC proliferation and confers resistance to cisplatin-induced TEC apoptosis. Our in vivo and in vitro findings in mice and humans clearly indicate that the CSF-1/CSF-1R mediates tubular self repair and self protection by promoting autocrine/ paracrine TEC proliferation and countering TEC apoptosis.

CSF-1 lessened interstitial fibrosis following I/R. The pathogenesis of interstitial fibrosis is controversial. One theory is that toxic damage to kidney tubules triggers interstitial fibrosis (38). Damaged tubules either directly or indirectly cause the release of molecules that subsequently transform interstitial cells into myofibroblasts 
A CSF-1-mediated proliferation (in vitro)

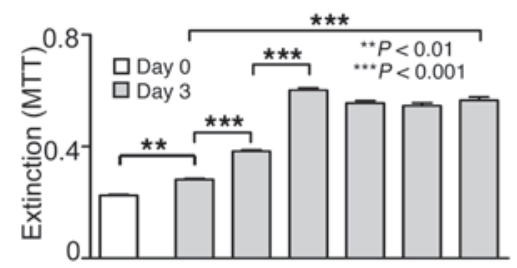

$\operatorname{CSF}-1(\mathrm{ng} / \mathrm{ml}) 0$
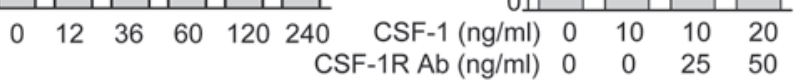

B CSF-1-mediated survival/proliferation (in vitro)

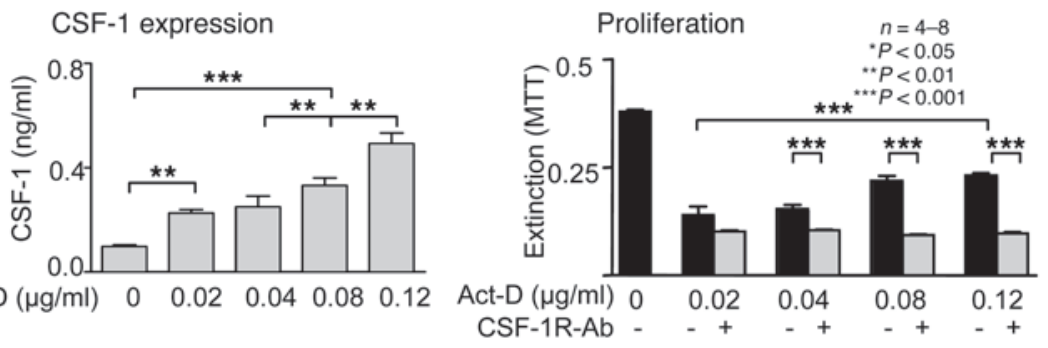

C CSF-1 autocrine proliferation (in vivo)

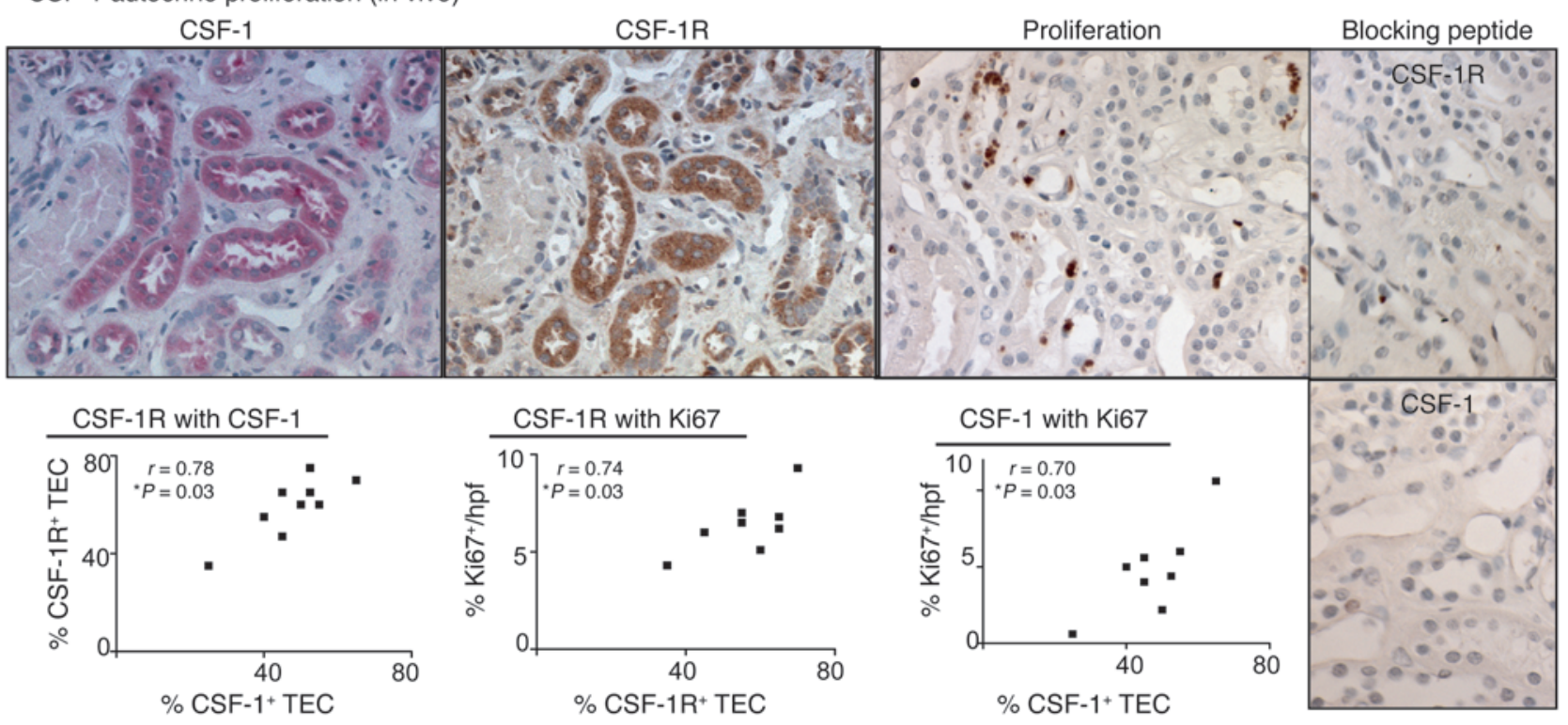

D CSF-1R phosphorylation

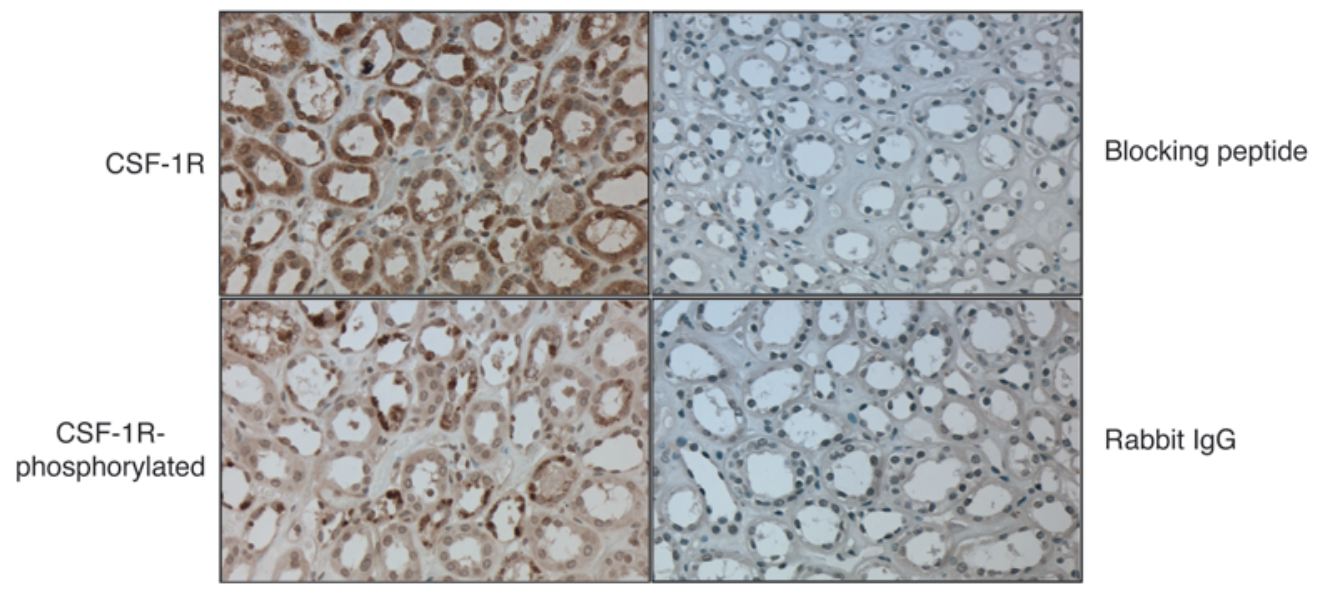




\section{Figure 5}

CSF-1 autocrine/paracrine regulated proliferation of human TECs. (A) Left panel: CSF-1 mediates HK2 TEC proliferation. CSF-1 stimulates proliferation of human HK2 TECs. Right panel: anti-CSF-1R Abs suppress CSF-1-mediated HK2 TEC proliferation. MTT assay. (B) Left panel: TEC injury increases TEC secretion of CSF-1. Following exposure of cultured HK2 TECs to increasing concentrations of actinomycin D (act-D) for 72 hours, we analyzed CSF-1 in the supernatant by ELISA. Right panel: anti-CSF-1R Abs inhibit TEC survival/proliferation in response to TEC injury. HK2 TECs were incubated with the indicated concentrations of actinomycin $\mathrm{D}$ in the presence and absence of anti-CSF-1R Abs for 72 hours prior to determination of cell mass by MTT assay. (C) CSF-1 expression, CSF-1R expression, and proliferation of TECs correlate in human transplants with tubular injury. Using serial sections of kidney biopsy specimens from patients with tubular injury and impaired renal function, we probed for CSF-1, CSF-1R, and proliferation in tubules. CSF-1R and CSF-1 are coexpressed on TECs by immunostaining. Note proliferating Ki67+ TECs in tubules coexpressing CSF-1 and CSF-1R. Right panels: CSF-1R and CSF-1 $A b$ specificity was verified using peptide preabsorption. Representative photomicrographs and correlation graphs. Original magnification, $\times 20$. Data show means \pm SEM. $n=8$ per group. (D) CSF-1R is tyrosine phosphorylated in human transplants with tubular injury. Using serial sections of kidney biopsy specimens, we probed for CSF-1R and CSF-1R phosphorylation at Y723. CSF-1R and tyrosine-phosphorylated CSF-1R are coexpressed on TECs by immunostaining. CSF-1R and phosphoY723 CSF-1R Ab specificity was verified using peptide preabsorption and rabbit lgG, respectively. Original magnification, $\times 40$.

(39). These myofibroblasts in turn generate collagen. Our findings indicate that macrophage-dependent and -independent CSF-1mediated mechanisms suppress interstitial fibrosis, since blocking the CSF-1R increases fibrosis far more than eliminating macrophages alone. This implies that events dependent on signaling via the CSF-1R on TECs may release molecules that suppress fibrosis. This intriguing concept will require further studies to identify CSF-1R $\mathrm{R}^{+}$ TEC mechanisms linked to renal interstitial fibrosis.

In conclusion, CSF-1 mediates renal repair after ischemic injury. While macrophages contribute to CSF-1-dependent renal repair, we have uncovered a CSF-1-dependent tubular autocrine/paracrine mechanism that aids in tubular regeneration and counters tubular destruction following acute renal injury. This finding has potential therapeutic application for a broad range of kidney diseases.

\section{Methods}

\section{Mice}

We purchased B6, C3H/Fej, and MRL/MpJ-Fas ${ }^{l p r} / F_{s} s^{l p r}\left(\mathrm{MRL}-\right.$ Fas $\left.^{l p r}\right)$ mice from The Jackson Laboratory. Csf1 $r^{+/-}$mice on the C3/B6 background (40) and transgenic mice (FVB) expressing lacZ under the control of CSF-1 promoter and the first intron $[\operatorname{TgN}(\mathrm{Csf1}-Z)$ Ers7/++ , referred to as $\operatorname{Tg} Z$ mice (27), were developed in the specific pathogen-free animal facility of the Albert Einstein College of Medicine. Transgenic mice $(\mathrm{B} 6 \times \mathrm{CBAF} 1)$ expressing EGFP under the control of the CSF-1R (c-fms) promoter and first intron (Tgfms-EGFP), referred to as MacGreen mice (FVB/B6 mixed background), were provided by D.A. Hume, University of Edinburgh, Edinburgh, Scotland (25). We backcrossed the TgZ and MacGreen mice onto the $\mathrm{B} 6$ background for 7 generations. These mice are referred to as MacGreen;B6 and TgZ;B6, respectively. We also used transgenic Tg(ITGAMDTR/EGFP)34Lan mice (FVB background), referred to as CD11b-DTR mice (The Jackson Laboratory) with a DT-inducible system that transient- ly depletes macrophages. Mice were housed and bred at Harvard Medical School. Use of mice in this study was reviewed and approved by the Standing Committee on Animals in the Harvard Medical School in adherence to standards set in the Guide for the care and use of laboratory animals (NIH publication no. 86-23. Revised 1996).

\section{$I / R$}

We anesthetized mice and exposed the left kidney through a flank incision. We induced ischemia by clamping the renal pedicle with nontraumatic microaneurysm clamps (Roboz). We removed the clamps after 30 minutes. The body temperature was controlled at $36.6-37.5^{\circ} \mathrm{C}$ throughout the procedure. We removed the obstructed kidney and fixed, sectioned, and analyzed tissue as previously described (26). Male mice were exclusively used for I/R experiments.

\section{Ab/cytokine administration}

We injected mice (i.p.) every 12 hours with purified recombinant human CSF-1 (50 $\mu \mathrm{g} / \mathrm{kg} /$ body weight) or daily with CSF-1R Abs (AFS98, $50 \mathrm{mg} / \mathrm{kg} /$ body weight; rat monoclonal anti-mouse Abs) (41) beginning at 1.5 days after $\mathrm{I} / \mathrm{R}$ and ending at various time points during I/R. CSF-1 was a gift from Chiron, and the AFS98 hybridoma was a gift of Shin-Ichi Nishikawa (RIKEN Center for Developmental Biology, Kobe, Japan). Mice receiving PBS and rat IgG were used as controls for CSF-1- and CSF-1R $\mathrm{Ab}$-injected mice, respectively.

\section{Renal histopathology}

We fixed kidneys in $10 \%$ neutral buffered formalin, embedded them in paraffin, and stained paraffin sections $(4 \mu \mathrm{m})$ with periodic acid-Schiff reagent. Kidney pathology was assessed as previously described (42).

\section{Collagen detection}

We stained paraffin sections after rehydration in picrosirius red solution for 1 hour and rinsed $(\times 2)$ with acidified water. We dehydrated and mounted the sections and analyzed the amount of stain using a Nikon Eclipse E1000 upright fluorescence microscope and Adobe Photoshop CS2.

\section{Renal function}

We measured BUN and albuminuria as previously described (42).

\section{Isolation of primary TECs}

We isolated and cultured TECs derived from B6 mice as previously reported (43).

\section{Proliferation}

Immunobistochemistry. We stained paraffin sections using a primary $\mathrm{Ab}$ against rabbit anti-human/mouse Ki67 (SP6; Lab Vision, Thermo Scientific) to identify proliferating TECs.

MTT assay. We cultured HK2 cells and primary isolated TECs (B6) in 96-well plates $\left(5 \times 10^{3}\right.$ cells/well) for 12 hours and stimulated these cells for 72 hours with human recombinant CSF-1 at various concentrations (12, $36,60,120$, and $240 \mathrm{ng} / \mathrm{ml})$. To verify specificity by blocking the CSF-1R, we stimulated cells with CSF-1 (10 and $20 \mathrm{ng} / \mathrm{ml})$ in combination with CSF-1R Abs (25 and $50 \mathrm{ng} / \mathrm{ml}$; Santa Cruz Biotechnology Inc.) for 72 hours. Furthermore, we stimulated cultured HK2 cells, primary mouse TECs with varying concentrations of human recombinant CSF-1 $(5,20$, 39 , and $78 \mathrm{ng} / \mathrm{ml})$ in combination with actinomycin $\mathrm{D}(0.02,0.04,0.08$, and $0.12 \mu \mathrm{g} / \mathrm{ml})$, cisplatin $(1 \mu \mathrm{g} / \mathrm{ml})$ or TNF- $\alpha(3,6,15$, and $30 \mathrm{ng} / \mathrm{ml})$, and LPS $(6.3,12.5,25$, and $50 \mathrm{ng} / \mathrm{ml})$ (Sigma-Aldrich). We analyzed proliferation using the MTT colorimetric assay (Roche) according to the manufacturer's instructions. 
A

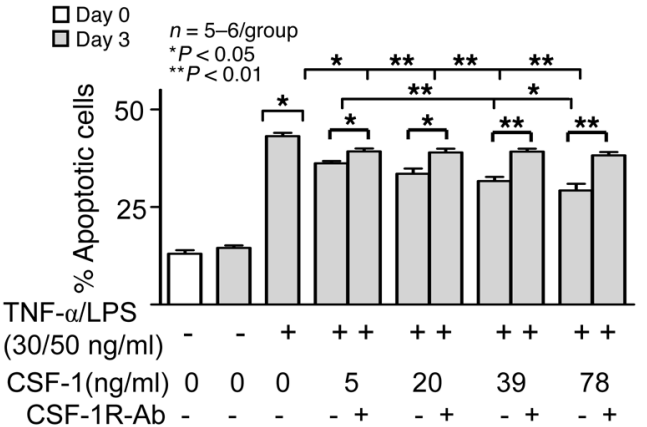

B

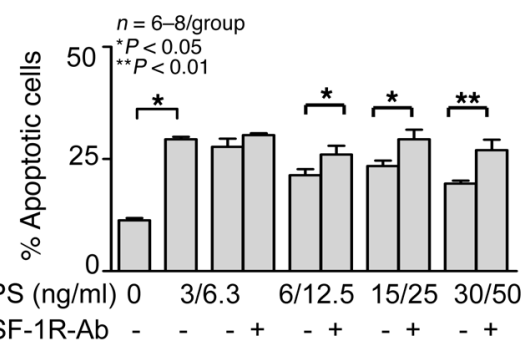

Figure 6

CSF-1 protects TECs by dampening apoptosis following injury. (A) CSF-1 suppresses TEC apoptosis induced by a 72-hour treatment of TECs with TNF- $\alpha /$ LPS. CSF-1 reduces TNF- $\alpha /$ LPS-induced TEC apoptosis assessed by flow cytometric annexin-PI analysis. Blocking the CSF-1R prevents CSF-1-mediated reduction in the number of apoptotic TECs. (B) TNF- $\alpha$ /LPS-injured TECs are self protective through a CSF-1-dependent mechanism that dampens TEC apoptosis. H2K cells were cultured for 72 hours in the absence of CSF-1. Blocking the CSF-1R increases TNF- $\alpha /$ LPS-induced TEC apoptosis. Data show means \pm SEM. $n=5-6$ per group.

\section{Cell lines}

Cell lines were used and cultured as previously described. Mouse cell lines were as follows: immortalized proximal TEC line (C1) (44), CD4 $4^{+} \mathrm{T}$ cell line (DO11.10) (45), and monocytic-macrophage cell line (RAW 264.7) (46). Human cell lines were as follows: immortalized proximal TEC line (HK2) (47), leukemic monocyte lymphoma cell line (U937) (48), erythromyeloblastoid leukemia cell line (K562) (49), promyelocytic leukemia cell line (HL60) (50), and immortalized T cell line (Jurkat) (51).

\section{CSF-1R transcript expression}

We analyzed the CSF-1R expression in primary TECs, BM macrophages, and cell lines using real-time, 2-step, quantitative PCR as previously described (RTQ-PCR) (52). The mRNA levels were normalized to those of GAPDH. We used the following PCR primers: mouse: GAPDH; sense, 5'-CATGGCCTCCAAGGAGTAAG-3', antisense, 5'-CCTAGGCCCCTCCTGTTATT-3'; and CSF-1R; sense 5'-CTACTGCTGTTGCTGCTCTTGT-3', anti-sense $5^{\prime}$-CGGCTCCTAGAGTCTTACCAAA-3'; and human: GAPDH; sense, 5'-CCCTCAACGACCACTTTGTCA-3', antisense, 5'-TTCCTCTTGTGCTCTTGCTGG-3'; and CSF-1R; sense 5'-TGAGCAAGACCTGGACAAGGA-3', antisense 5'-CCATTGGTCAACAGCACGTTA-3'.

\section{Immunoprecipitation and Western blot}

Cell lines, primary isolated TECs, and BM macrophages grown on $10-\mathrm{cm}$ plates were collected when confluent. Stimulation with TPA $(1 \mu \mathrm{M})$ was done for 24 hours in the human cell line, followed by stimulation with CSF-1 (100 ng/ml) for 1, 3, 5, and 10 minutes. Primary isolated TECs and
BM macrophages were stimulated with CSF-1 (100 ng/ml) for 1, 3, 5, and 10 minutes. We resuspended the cell pellets in lysis buffer (2\% NP-40, $10 \mathrm{mM}$ Tris- $\mathrm{HCl}, 50 \mathrm{mM} \mathrm{NaCl}, 30 \mathrm{mM} \mathrm{Na}{ }_{4} \mathrm{P}_{2} \mathrm{O}_{7}, 50 \mathrm{mM} \mathrm{NaF}, 1 \mathrm{mM}$ $\mathrm{Na}_{3} \mathrm{VO}_{4}, 5 \mu \mathrm{MZnCl}_{2}, 1 \mathrm{mM}$ benzamidine, $20 \mu \mathrm{g} / \mathrm{ml}$ leupeptin, and $10 \mu \mathrm{g} / \mathrm{ml}$ aprotinin, $\mathrm{pH}$ 7.2). We lysed cells by successive freezing and thawing in liquid nitrogen $(\times 3)$. Cell lysates were passed through a 25 -gauge needle $(\times 5)$ followed by centrifugation for 20 minutes at $10,000 \mathrm{~g}$, and supernatants were stored at $-80^{\circ} \mathrm{C}$ until analysis. For immunoprecipitation, NP-40 lysates were incubated with $5 \mu \mathrm{g}$ of rabbit anti-mouse CSF-1R $\mathrm{C}$-terminal domain peptide Abs (C15; ref. 53) and $20 \mu \mathrm{l}$ protein A-sepharose $4 \mathrm{~B}$ beads (Zymed) overnight at $4{ }^{\circ} \mathrm{C}$ with continuous mixing and then centrifuged at $13,000 \mathrm{~g}$ at $4^{\circ} \mathrm{C}$ for 30 seconds. The supernatant was removed, and the beads were washed at $4^{\circ} \mathrm{C}$ with $1 \mathrm{ml}$ wash buffer (lysis buffer containing $0.5 \%$ NP-40 without leupeptin and aprotinin) 5 times and $200 \mu \mathrm{l}$ double-distilled water once; proteins were eluted with $10 \mu \mathrm{l}$ $3 \%$ SDS sample buffer at $65^{\circ} \mathrm{C}$ for 10 minutes. SDS-PAGE (10\% acrylamide) and Western blotting with rabbit anti-mouse-CSF-1R IK Abs (54) were carried out as previously described (53).

\section{CSF-1R and CSF-1 expression}

Immunofluorescence: in vitro. We isolated and cultured TECs and BM macrophages from B6 mice as previously described $(43,55)$. Cells $\left(1 \times 10^{4} /\right.$ well $)$ were stimulated with mouse CSF-1 (10 ng/ml; PeproTech) for 48 hours. We fixed cells with methanol for 5 minutes on coverslips and incubated them with rabbit anti-mouse CSF-1R IK Abs (generated by R. Stanley) for 1 hour at RT. We detected CSF-1R by incubating cells with biotinylated goat-anti-rabbit Abs (Vector Laboratories) for 1 hour, followed by streptavidin-FITC (eBioscience) for 30 minutes. We mounted the coverslips with Vectashield (Vector Laboratories) and analyzed them using a Nikon Eclipse E1000 upright fluorescence microscope.

Immunofluorescence: in vivo. To identify CSF-1R-bearing cells and CSF-1 expression in the kidney, we fixed, prepared, and analyzed kidneys from the mutant MacGreen and $\mathrm{TgZ}$ reporter gene mice, respectively, as previously described $(25,26)$.

Immunohistochemistry: paraffin sections. We deparaffinized and rehydrated serial sections $(4 \mu \mathrm{m})$. Antigen retrieval was performed by immersion in citrate buffer ( $\mathrm{pH} 6.0$ ) at $95^{\circ} \mathrm{C}$ for 45 minutes. Endogenous peroxidase activity was blocked by incubation with $3 \% \mathrm{H}_{2} \mathrm{O}_{2}$ in methanol. We blocked the nonspecific binding of avidin and biotin using an avidin/biotin blocking kit according to the manufacturer's instructions (Vector Laboratories). After we blocked the nonspecific binding of goat or rabbit Abs by incubating with $10 \%$ normal goat or rabbit serum, respectively, we incubated the sections with either goat anti-human CSF-1 Abs (N-16; Santa Cruz Biotechnology Inc.) or rabbit anti-human phospho-M-CSFR Tyr 723 Abs (49C10; Cell Signaling Technology) at $4{ }^{\circ} \mathrm{C}$ overnight. We detected these primary Abs by incubation with biotinylated rabbit anti-goat $\mathrm{Abs}$, followed by peroxidase-conjugated $\mathrm{ABC}$ solution and development with 3'3-diaminobenzidine (Vector Laboratories) (CSF-1) or by use of the EnVision $^{+}$System (Dako) for rabbit primary Abs according to the manufacturer's instructions. Sections were counterstained with Mayer's Hematoxylin (Sigma-Aldrich). We confirmed specificity by using specific blocking peptides for CSF-1 and CSF-1R (Santa Cruz Biotechnology Inc.) and replacing the primary Abs with goat IgG for CSF-1 and rabbit IgG (eBioscience) for the other Abs. We determined the number of positive cells in 10 randomly selected high-power fields and evaluated the corresponding areas within serial sections for the correlation analysis.

Immunohistochemistry: frozen sections. We stained frozen kidney sections $(4 \mu \mathrm{m})$ for the presence of macrophages and $\mathrm{T}$ cell populations, using anti-mouse CD68 Abs (FA-11; AbD Serotec), anti-mouse CD4 Abs (RM 4-5), and anti-mouse CD8 Abs (53-6.7) (eBioscience), respectively (56). 
We identified apoptotic cells using anti-cleaved caspase-3 (Asp175) (Cell Signaling Technology) (56).

\section{KIM-1 expression}

Cryosections $(7 \mu \mathrm{m})$ were mounted on Fisher Superfrost Plus (Fisher Scientific) microscope slides, air-dried, and treated for immunofluorescence using standard techniques. The polyclonal anti-KIM-l antisera R9 was used at a 1:200 dilution (21). Cy3-conjugated anti-rabbit secondary Abs were from Dako. Sections were mounted in Vectashield containing 4',6-diamino-2-phenylindole (Vector Laboratories). Confocal immunofluorescent images were obtained using a Nikon C1 D-Eclipse confocal microscope. Projection images were generated from $8 \mathrm{Z}$-stack images acquired at $0.1 \mu \mathrm{m}$ steps. To allow comparison among sections, all confocal settings were kept constant among sections.

\begin{abstract}
Apoptosis
We cultured human HK2 cells and primary mouse TECs (B6) in 6-well (apoptosis assay) or 96-well (proliferation assay) plates $\left(5 \times 10^{4} /\right.$ well) for 12 hours and stimulated these cells with varying concentrations of human recombinant CSF-1 $(5,20,39$, and $78 \mathrm{ng} / \mathrm{ml})$ in combination with actinomycin D $(0.05 \mu \mathrm{g} / \mathrm{ml})$, cisplatin $(1 \mu \mathrm{g} / \mathrm{ml})$ or TNF- $\alpha(3,6,15$, and $30 \mathrm{ng} / \mathrm{ml})$, and LPS $(6.3,12.5,25$, and $50 \mathrm{ng} / \mathrm{ml})$. After 72 hours, we assessed apoptosis by flow cytometry using an annexin V-FITC-PI kit (BD Biosciences) according to the manufacturer's instructions.
\end{abstract}

\section{Renal biopsy specimens}

Biopsy sections (discarded tissues) from kidney transplants were provided by the Department of Pathology (J. Kriegsmann), Johannes-Gutenberg University. Kidneys were biopsied between 0 and 2 months after engraftment due to impaired renal function. The tubules were abnormal in these biopsies and included at least one of the following: dilatation, vacuolariza- tion, hyaline casts, and/or atrophy. Leukocytes were noted in the interstitium, and there was a mild increase in mesangial matrix within glomeruli.

\section{CSF-1 ELISA}

To quantify the levels of CSF-1 in supernatants, we evaluated samples using an ELISA as previously described (57).

\section{Deleting macrophages}

To delete macrophages, we injected CD11b-DTR mice i.v. with DT (SigmaAldrich) at $20 \mathrm{ng} / \mathrm{g}$ body weight.

\section{Statistics}

Data represent the mean \pm SEM and were prepared using GraphPad Prism software, version 4.0. We used the nonparametric Mann-Whitney $U$ test to evaluate $P$ values. $P<0.05$ was considered significant. For correlation analysis, we used Spearman's correlation calculation.

\section{Acknowledgments}

This work was supported by NIH grants DK 36149 (to V.R. Kelley), CA32551 (to E.R. Stanley), and CA26504 (to E.R. Stanley). J. Menke is supported by the Deutsche Forschungsgemeinschaft (ME-3194/1-1). A. Schwarting is supported by the Deutsche Forschungsgemeinschaft (Schw785/2-1).

Received for publication March 3, 2009, and accepted in revised form May 6, 2009.

Address correspondence to: Vicki Rubin Kelley, Harvard Institutes of Medicine, 4 Black Fan Circle, Boston, Massachusetts 02115, USA. Phone: (617) 525-5915; Fax: (617) 525-5830; E-mail: vkelley@ rics.bwh.harvard.edu.
1. Humphreys, B.D., et al. 2008. Intrinsic epithelial cells repair the kidney after injury. Cell Stem Cell. 2:284-291

2. Goligorsky, M.S. 2008. Immune system in renal injury and repair: burning the candle from both ends? Pharmacol. Res. 58:122-128.

3. Witzgall, R., Brown, D., Schwarz, C., and Bonventre, J.V. 1994. Localization of proliferating cell nuclear antigen, vimentin, c-Fos, and clusterin in the postischemic kidney. Evidence for a heterogenous genetic response among nephron segments, and a large pool of mitotically active and dedifferentiated cells. J. Clin. Invest. 93:2175-2188.

4. Thadhani, R., Pascual, M., and Bonventre, J.V. 1996. Acute renal failure. N. Engl. J. Med. 334:1448-1460.

5. Bonventre, J.V., and Weinberg, J.M. 2003. Recent advances in the pathophysiology of ischemic acute renal failure. J. Am. Soc. Nephrol. 14:2199-2210.

6. Bonventre, J.V., and Zuk, A. 2004. Ischemic acute renal failure: an inflammatory disease? Kidney Int. 66:480-485.

7. Duffield, J.S., and Bonventre, J.V. 2005. Kidney tubular epithelium is restored without replacement with bone marrow-derived cells during repair after ischemic injury. Kidney Int. 68:1956-1961.

8. Pixley, F.J., and Stanley, E.R. 2004. CSF-1 regulation of the wandering macrophage: complexity in action. Trends Cell Biol. 14:628-638.

9. Chitu, V., and Stanley, E.R. 2006. Colony-stimulating factor- 1 in immunity and inflammation. Curr. Opin. Immunol. 18:39-48.

10. Lenda, D.M., Kikawada, E., Stanley, E.R., and Kelley, V.R. 2003. Reduced macrophage recruitment, proliferation, and activation in colony-stimulating factor-1-deficient mice results in decreased tubular apoptosis during renal inflammation. J. Immunol. 170:3254-3262.
11. Lenda, D.M., Stanley, E.R., and Kelley, V.R. 2004. Negative role of colony-stimulating factor- 1 in macrophage, $\mathrm{T}$ cell, and $\mathrm{B}$ cell mediated autoimmune disease in MRL-Fas(lpr) mice. J. Immunol. 173:4744-4754.

12. Aharinejad, S., et al. 2008. Colony-stimulating factor- 1 transfection of myoblasts improves the repair of failing myocardium following autologous myoblast transplantation. Cardiovasc. Res. 79:395-404.

13. Ricardo, S.D., van Goor, H., and Eddy, A.A. 2008. Macrophage diversity in renal injury and repair. J. Clin. Invest. 118:3522-3530.

14. Gratchev, A., et al. 2006. Mphi1 and Mphi2 can be repolarized by Th 2 or Th 1 cytokines, respectively, and respond to exogenous danger signals. Immunobiology. 211:473-486.

15. Sapi, E. 2004. The role of CSF-1 in normal physiology of mammary gland and breast cancer: an update. Exp. Biol. Med. (Maywood). 229:1-11.

16. Borycki, A.G., Smadja, F., Stanley, R., and Leibovitch, S.A. 1995. Colony-stimulating factor 1 (CSF-1) is involved in an autocrine growth control of rat myogenic cells. Exp. Cell. Res. 218:213-222.

17. Sapi, E., Flick, M.B., Rodov, S., Carter, D., and Kacinski, B.M. 1998. Expression of CSF-I and CSF-I receptor by normal lactating mammary epithelial cells. J. Soc. Gynecol. Investig. 5:94-101.

18. Yui, M.A., Brissette, W.H., Brennan, D.C., Wuthrich, R.P., and Rubin-Kelley, V.E. 1991. Increased macrophage colony-stimulating factor in neonatal and adult autoimmune MRL-lpr mice. Am. J. Pathol. 139:255-261.

19. Bloom, R.D., Florquin, S., Singer, G.G., Brennan, D.C., and Kelley, V.R. 1993. Colony stimulating factor- 1 in the induction of lupus nephritis. Kidney Int. 43:1000-1009.

20. Naito, T., et al. 1996. Macrophage growth factors introduced into the kidney initiate renal injury. Mol. Med. 2:297-312.

21. Ichimura, T., et al. 1998. Kidney injury molecule-1 (KIM-1), a putative epithelial cell adhesion molecule containing a novel immunoglobulin domain, is up-regulated in renal cells after injury. J. Biol. Chem. 273:4135-4142.

22. Bonventre, J.V. 2003. Dedifferentiation and proliferation of surviving epithelial cells in acute renal failure. J. Am. Soc. Nephrol. 14(Suppl. 1):S55-S61.

23. Hume, D.A. 2008. Differentiation and heterogeneity in the mononuclear phagocyte system. Mucosal Immunol. 1:432-441.

24. Duffield, J.S., et al. 2005. Selective depletion of macrophages reveals distinct, opposing roles during liver injury and repair. J. Clin. Invest. 115:56-65.

25. Sasmono, R.T., et al. 2003. A macrophage colonystimulating factor receptor-green fluorescent protein transgene is expressed throughout the mononuclear phagocyte system of the mouse. Blood. 101:1155-1163

26. Jang, M.H., et al. 2006. Distinct in vivo roles of colonystimulating factor- 1 isoforms in renal inflammation. J. Immunol. 177:4055-4063.

27. Ryan, G.R., et al. 2001. Rescue of the colonystimulating factor 1 (CSF-1)-nullizygous mouse (Csf1(op)/Csf1(op)) phenotype with a CSF-1 transgene and identification of sites of local CSF-1 synthesis. Blood. 98:74-84.

28. Liu, K.D., and Brakeman, P.R. 2008. Renal repair and recovery. Crit. Care Med. 36:S187-S192.

29. van Amerongen, M.J., et al. 2007. Macrophage depletion impairs wound healing and increases left ventricular remodeling after myocardial injury in mice. Am. J. Pathol. 170:818-829.

30. Arnold, L., et al. 2007. Inflammatory monocytes recruited after skeletal muscle injury switch into 
antiinflammatory macrophages to support myogenesis. J. Exp. Med. 204:1057-1069.

31. Nahrendorf, M., et al. 2007. The healing myocardium sequentially mobilizes two monocyte subsets with divergent and complementary functions. J. Exp. Med. 204:3037-3047.

32. Jang, H.S., Kim, J., Park, Y.K., and Park, K.M. 2008. Infiltrated macrophages contribute to recovery after ischemic injury but not to ischemic preconditioning in kidneys. Transplantation 85:447-455.

33. Ide, H., et al. 2002. Expression of colony-stimulating factor 1 receptor during prostate development and prostate cancer progression. Proc. Natl. Acad. Sci. U. S. A. 99:14404-14409.

34. Kacinski, B.M. 1997. CSF-1 and its receptor in breast carcinomas and neoplasms of the female reproductive tract. Mol. Reprod. Dev. 46:71-74.

35. Sakai, M., et al. 1997. Production of heparin binding epidermal growth factor-like growth factor in the early phase of regeneration after acute renal injury. Isolation and localization of bioactive molecules. J. Clin. Invest. 99:2128-2138.

36. Homma, T., et al. 1995. Induction of heparin-binding epidermal growth factor-like growth factor mRNA in rat kidney after acute injury. J. Clin. Invest. 96:1018-1025.

37. Cantaluppi, V., et al. 2008. Macrophage stimulating protein may promote tubular regeneration after acute injury. J. Am. Soc. Nephrol. 19:1904-1918.

38. Christensen, E.I., and Verroust, P.J. 2008. Interstitial fibrosis: tubular hypothesis versus glomerular hypothesis. Kidney Int. 74:1233-1236.

39. Iwano, M., and Neilson, E.G. 2004. Mechanisms of tubulointerstitial fibrosis. Curr. Opin. Nephrol. Hypertens. 13:279-284.

40. Dai, X.M., Zong, X.H., Akhter, M.P., and Stanley,
E.R. 2004. Osteoclast deficiency results in disorganized matrix, reduced mineralization, and abnormal osteoblast behavior in developing bone. J. Bone Miner. Res. 19:1441-1451.

41. Sudo, T., et al. 1995. Functional hierarchy of c-kit and c-fms in intramarrow production of CFU-M. Oncogene. 11:2469-2476.

42. Menke, J., et al. 2007. Programmed death 1 ligand (PD-L) 1 and PD-L2 limit autoimmune kidney disease: distinct roles. J. Immunol. 179:7466-7477.

43. Faust, J., et al. 2002. Correlation of renal tubular epithelial cell-derived interleukin-18 up- regulation with disease activity in MRL-Faslpr mice with autoimmune lupus nephritis. Arthritis Rheum. 46:3083-3095

44. Wuthrich, R.P., et al. 1990. MHC class II, antigen presentation and tumor necrosis factor in renal tubular epithelial cells. Kidney Int. 37:783-792.

45. Huang, J., Tatsumi, T., Pizzoferrato, E., Vujanovic, N., and Storkus, W.J. 2005. Nitric oxide sensitizes tumor cells to dendritic cell-mediated apoptosis, uptake, and cross-presentation. Cancer Res. 65:8461-8470.

46. Ramamoorthy, L., and Tizard, I.R. 1998. Induction of apoptosis in a macrophage cell line RAW 264.7 by acemannan, a beta- $(1,4)$-acetylated mannan. Mol. Pharmacol. 53:415-421.

47. Lee, H.T., and Emala, C.W. 2002. Adenosine attenuates oxidant injury in human proximal tubular cells via A1 and A2a adenosine receptors10.1152/ ajprenal.00195.2001. Am. J. Physiol. Renal Physiol. 282:F844-F852.

48. Strefford, J.C., et al. 2001. The characterisation of the lymphoma cell line U937, using comparative genomic hybridisation and multi-plex FISH. Cytogenet. Cell Genet. 94:9-14.
49. Fordis, C.M., Anagnou, N.P., Dean, A., Nienhuis, A.W., and Schechter, A.N. 1984. A \{beta\} -globin gene, inactive in the K562 leukemic cell, functions normally in a heterologous expression system. Proc. Natl. Acad. Sci. U. S. A. 81:4485-4489.

50. Bar-Shavit, Z., et al. 1985. Differentiation of a human leukemia cell line and expression of collagenase inhibitor. Proc. Natl. Acad. Sci. U. S. A. 82:5380-5384.

51. Myrick, D., et al. 1999. Paclitaxel-induced apoptosis in Jurkat, a leukemic T cell line, is enhanced by ceramide. Leuk. Res. 23:569-578.

52. Kikawada, E., Lenda, D.M., and Kelley, V.R. 2003. IL-12 Deficiency in MRL-Fas(lpr) mice delays nephritis and intrarenal IFN-gamma expression, and diminishes systemic pathology. J. Immunol. 170:3915-3925.

53. Yu, W., et al. 2008. CSF-1 receptor structure/function in MacCsf1 $\mathrm{r}^{-/-}$macrophages: regulation of proliferation, differentiation, and morphology. J. Lenkoc. Biol. 84:852-863.

54. Yeung, Y.G., Wang, Y., Einstein, D.B., Lee, P.S., and Stanley, E.R. 1998. Colony-stimulating factor-1 stimulates the formation of multimeric cytosolic complexes of signaling proteins and cytoskeletal components in macrophages. J. Biol. Chem. 273:17128-17137.

55. Stanley, E.R. 1985. The macrophage colony-stimulating factor, CSF-1. Methods Enzymol. 116:564-587.

56. Moore, K.J., Yeh, K., Naito, T., and Kelley, V.R. 1996. TNF-alpha enhances colony-stimulating factor-1induced macrophage accumulation in autoimmune renal disease. J. Immunol. 157:427-432.

57. Menke, J., et al. 2008. Sunlight triggers cutaneous lupus through a CSF-1-dependent mechanism in MRL-Fas(lpr) mice. J. Immunol. 181:7367-7379. 